\title{
EVOLUCIÓN DE FASES MAGNÉTICAS PRESENTES EN ARCILLAS DE BOYACÁ SOMETIDAS A ALTAS TEMPERATURAS
}

\section{(Evolution of magnetic phases located in Boyacá clays subject to high temperatures)}

\author{
Oscar Orlando Ruge Guerrero ${ }^{1}$, Segundo Agustín Martínez Ovalle ${ }^{1}$, Mercedes Díaz Lagos² \\ ${ }^{1}$ Universidad Pedagógica y Tecnológica de Colombia, (Tunja - Boyacá, Colombia). Grupo de Física Nuclear \\ Aplicada y Simulación. \\ Escuela de Ingeniería Geológica, Universidad Pedagógica y Tecnológica de Colombia, (Sogamoso - Boyacá, \\ Colombia)
}

(Recibido abril 10 de 2016 y aceptado junio 13 de 2016)

\begin{abstract}
Resumen
En este trabajo analizamos muestras de arcilla obtenidas de la región de Tunja - Boyacá, con el objetivo de estudiar el efecto de la temperatura de calentamiento en el color de estos materiales y a la vez como indicativo de la naturaleza y la distribución de sus constituyentes de óxidos de hierro $\mathrm{Fe}_{2} \mathrm{O}_{3}$. Estos colores son de relevancia estética en la fabricación de cubiertas y ladrillos en construcción de viviendas. Para evaluar la evolución de las fases, su composición y microestructura en las muestras se utilizó Fluorescencia de rayos X (XRF), Difracción de rayos X (DRX), Espectroscopia Mössbauer (EM) y Microscopia electrónica de barrido (SEM) para muestras sometidas a tratamiento térmico entre $\left(400-1200{ }^{\circ} \mathrm{C}\right)$. Los resultados indican que las propiedades espectrales de las muestras están influenciadas principalmente por la mineralogía del Fe. Se identificaron fases de goetita y hematita en todas las muestras. Los análisis Mössbauer indicaron que con el incremento de la temperatura, la arcilla natural presenta un aumento en la fase de hematita que se refleja también en un incremento del tono rojo del cuerpo de la misma, en contraste con muestras de tono amarillo de la arcilla natural. La formación de hematita y el aumento en su ordenamiento magnético se debe en gran parte a la desaparición de la materia orgánica y la transformación de iones de hierro dispuestos en las fases mineralógicas de la arcilla natural.
\end{abstract}

Palabras clave: Espectroscopia Mössbauer, fases de hierro, hematita, calentamiento, mineralogía.

\begin{abstract}
In this paper we analyze clay samples obtained from the region of Tunja Boyacá to study the effect of heating temperature in the color of these materials and at the same time as indicative of the nature and distribution of constituents iron oxide $\mathrm{Fe}_{2} \mathrm{O}_{3}$. These colors are of decorative importance in the manufacture of roofing and brick housing construction. X-ray Fluorescence (XRF), X-ray Diffraction (XRD), Mössbauer Spectroscopy (MS) and Scanning Electron Microscopy (SEM) for samples subjected to heat treatment between $\left(400-1200^{\circ} \mathrm{C}\right)$ was used to assess the evolution of the phases and microstructure in the samples. The results indicate that the spectral properties of the samples are influenced primarily by the mineralogy of Fe. Goethite and hematite phases were identified in all samples. Mössbauer analyzes indicated that with increasing temperature of the raw clay presents an increase in phase of hematite and the red tone from the body of the same, in contrast to samples of yellow tone natural clay. Hematite formation and increased its magnetic ordering is largely due to the disappearance of organic matter and the oxidation of iron ions arranged in mineralogical phases of raw clay.
\end{abstract}

Keywords: Spectroscopy Mössbauer, phases of iron, hematite, heating, mineralogy. 


\section{INTRODUCCIÓN}

Los materiales arcillosos juegan un papel importante en los procesos geológicos y ambientales, ya que son componentes ubicuos de suelos, sedimentos y alteración hidrotermal de rocas. Además, son el principal ingrediente utilizado en la cerámica. Este material de grano fino presenta un comportamiento dúctil cuando se mezcla con agua. En la cocción de la arcilla para obtener los productos finales, los minerales de arcilla se someten a una secuencia de modificaciones químicas y estructurales (deshidratación, oxidación, deshidroxilación, descomposición y formación de nuevas fases) que generalmente mejoran su resistencia mecánica, durabilidad. La temperatura a la que se producen los cambios depende de la química y composición mineralógica de la arcilla original, la duración de la calcinación y la atmosfera en la que la calcinación se ha realizado, pueden influir en su uso tecnológico. Su geoquímica es compleja, pero por lo general se componen de caolinita (más de 70\%), muscovita-illita y óxidos de Fe. Estos minerales se utilizan tradicionalmente en la industria para la producción de baldosas (Kamseu et al, 2007; Njoya et al., 2010) y cerámicas refractarias (Djangang et al., 2008; 2011).

Se han utilizado para su caracterización diversas técnicas, no sólo para determinar su estructura cristalina, su estabilidad térmica, sino también cuantificar su concentración y obtener información sobre las fases de los minerales que contienen $\mathrm{Fe}$ (goethita, hematita y caolinita). En particular, para las arcillas de la región de Boyacá - Colombia; se han caracterizado en la mayoría de los casos utilizando tres técnicas complementarias, DRX, FRX y SEM (Naranjo et al., 2007) pero hasta el momento no se tiene reporte alguno sobre estudios realizados en arcillas mediante Espectroscopía Mössbauer-EM, la cual permite detectar la presencia de fases de Fe inducidas por efecto de la temperatura. A nivel mundial se han reportado diversos estudios sobre materiales arcillosos que muestran la eficacia de la EM para identificar óxidos, hidróxidos y otros minerales que contengan Fe en bajas concentraciones (Murad, 2006). En el trabajo de Chevalier et al., (1976), se muestra que los óxidos de Fe presentes en los minerales de la arcilla son los principales causantes del color de la misma y dicho color se hace más intenso a medida que la arcilla se somete a mayores temperaturas de calentamiento.
La Espectroscopía Mössbauer, es una técnica que se utiliza rutinariamente para la caracterización del estado de oxidación del $\mathrm{Fe}$ en arcillas y minerales de arcilla. En casos favorables se puede establecer la coordinación de $\mathrm{Fe}$ y las propiedades magnéticas inducidas por la presencia de Fe en la estructura. Las investigaciones que se han hecho mediante EM sobre arcillas o productos de estas, como los cerámicos muestran aportes significativos, un caso en particular fue explorado por Sherriff et al., (2002), quienes observaron detalladamente los cambios de color en la arcilla argumentando que la tonalidad rojiza que toman algunos cerámicos se debe al aumento de hematita a medida que se calienta dicha arcilla. Otras tonalidades como por ejemplo el color blanco se asocian a minerales de arcilla que no contienen óxidos de Fe (hematita, maghemita), pero si tienen incorporados cationes de Fe en fases de silicatos los cuales provocan la falta de color. De la misma forma, los tonos negros presentes en los bordes de algunos materiales cerámicos se deben a la presencia de maghemita en comparación a la hematita que podría asociarse principalmente a un bloqueo temporal de aire. El interés por conocer las diferentes tonalidades, producto de los minerales de arcilla es reportado por Hradil et al., (2003), quienes presentan una recopilación del aporte de la arcilla y los pigmentos de óxidos de Fe en la historia de la pintura.

Motivados por la mineralogía, las características geoquímicas y la aplicación de las arcillas de la región ladrillera de Boyacá nuestro objetivo fue evaluar su comportamiento al ser sometidas a diferentes temperaturas de calentamiento artificial principalmente en la identificación de las transformaciones térmicas de minerales de arcilla y óxidos de Fe.

\section{MATERIAL Y MÉTODO}

\subsection{Localización y Geología}

Las muestras estudiadas se tomaron de la región que se encuentra ubicada en la parte nororiental de la ciudad de Tunja, Boyacá, con coordenadas $5^{\circ} 33^{\prime} 38,9^{\prime \prime} \mathrm{N}$ $73^{\circ} 19^{\prime} 0,8^{\prime \prime} \mathrm{E}$ a una altura promedio de $2860 \mathrm{msnm}$. El terreno se caracteriza por ser bastante árido y despejado, provocando que la arcilla tenga contacto permanente con el ambiente, lo cual provoca los procesos de oxidación en algunos de los minerales presentes en la estructura de la arcilla natural. El lugar presenta estratificaciones de mantos de arcilla de color

Rev. 12+D. Vol.16 N² 2. Julio-Diciembre. 2016. Sogamoso-Boyacá, Colombia. 
marrón y rojizo en general. En la elección del lugar de muestreo se tuvo en cuenta la presencia de ladrilleras que ofrecen sus productos a la ciudad y región en general. La figura 1 muestra el mapa geológico de Tunja Boyacá, en el cual se amplía la zona de donde se extrajo la muestra representativa de arcilla natural.

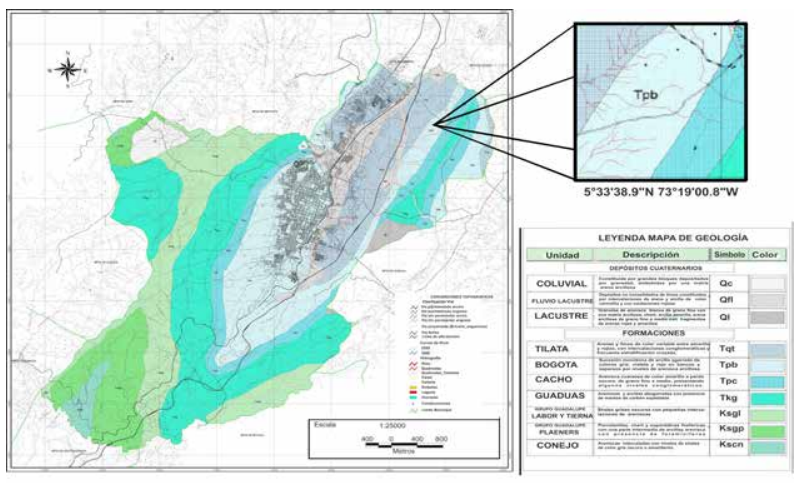

Figura 1. Mapa Geológico de Tunja Boyacá-Colombia y ubicación geoespacial del sector donde se tomó la muestra representativa de arcilla natural. [P02-mapa_geologia1. Planchas I. G. A. C. Mario López S. (1977-1979)]

\subsection{Preparación y calentamiento de las muestras}

Las muestras naturales se extrajeron mediante el socavado a una profundidad de $50 \mathrm{~cm}$, se tomó una muestra representativa de $25 \mathrm{~kg}$ la cual se secó a temperatura ambiente $\left(20 \pm 2^{\circ} \mathrm{C}\right)$ hasta peso constante. Posteriormente es llevada a molienda fina con mortero de Agata y tamizada hasta obtener una muestra homogénea de (<80 micras). Para estudiar la influencia de la temperatura de calcinación artificial en la evolución de la fase de Fe durante la calcinación de las arcillas se tomaron fracciones de la muestra representativa tamizada (en cantidades de $1 \mathrm{~g}$ ) y se colocaron en una mufla LABTECH referencia LEF605P-17 que alcanza una temperatura de $\approx 1500{ }^{\circ} \mathrm{C}$. Inicialmente se eleva la temperatura a una velocidad de $20^{\circ} \mathrm{C} / \mathrm{min}$ hasta llegar a $400^{\circ} \mathrm{C}$, una vez obtenida la temperatura deseada se mantiene la muestra durante 4 horas. Las muestras se dejaron enfriar en el horno hasta alcanzar la temperatura ambiente. El procedimiento se repite para otras fracciones de muestra representativa y para temperaturas de calcinación de $600^{\circ} \mathrm{C}, 800^{\circ} \mathrm{C}, 1000^{\circ} \mathrm{C}$ y $1200^{\circ} \mathrm{C}$.

\subsection{Caracterización de las muestras}

Las muestras se clasificaron de acuerdo con el sistema de color de Munsell que especifica el color basado en el matiz, luminosidad y pureza del color [17].

La composición elemental se determinó usando un espectrómetro de Fluorescencia de Rayos $X$ de energía dispersa PANalytical modelo Axios Petro de $4 \mathrm{~kW}$, con un detector de Xenón (Xe) de centelleo de flujo y ánodo de Rodio (Rh). El análisis cuantitativo de los elementos se obtuvo empleando el método de los parámetros fundamentales mediante el software del equipo OMNIAN.

Los minerales presentes en la muestra de arcilla natural sin calentar, fueron identificados mediante DRX utilizando un difractometro XPertPro PaNalytical con ánodo de $\mathrm{Cu}$, cuyo potencial del tubo es de $45 \mathrm{kV}$ y $40 \mathrm{~mA}$ con un detector Xcellerator y tamaño de paso de $0,0170^{\circ}$ con un tiempo de barrido de 36.1776 s, en configuración Bragg Bretano.

Los espectros Mössbauer de la arcilla natural sin calentar y calentadas artificialmente de 400 a $1200{ }^{\circ} \mathrm{C}$ se obtuvieron con un espectrómetro FAST en modo de transmisión en el Laboratorio de Radiaciones Nucleares de la Universidad Pedagógica y Tecnológica de Colombia. Este espectrómetro consta de una fuente de ${ }^{57} \mathrm{Co}$ embebida en una matriz de Rh dispuesta a una velocidad de $12 \mathrm{~mm} \cdot \mathrm{s}^{-1}$, los fotones transmitidos se detectaron mediante un contador proporcional LND con gas de Kriptón, a un voltaje de trabajo de 1800 V. Los valores del corrimiento isomérico (IS) se reportan con respecto al IS del calibrado de Fe puro al 90\%. Para el ajuste de los espectros se usó el software MOSFIT. Para la identificación de los minerales de Fe presentes en la arcilla fue necesario la guía del Mossbauer Mineral HandBook (2005).

Para las imágenes de SEM, se utilizó el microscopio marca ZEISS, con un aumento de 200X Instituto para la Investigación e Innovación en Ciencia y Tecnología de Materiales de la Universidad Pedagógica y Tecnológica de Colombia. 


\section{ANALISIS Y RESULTADOS}

\subsection{Identificación de fases mineralógicas de la arcilla natural}

La composición química de la arcilla natural se presenta en la Tabla 1. Esta composición está dada en forma de óxido y expresada en porcentaje en peso

Tabla 1. Composición elemental de la arcilla natural en orden creciente de concentración de especies obtenidas mediante Fluorescencia de Rayos X.

\begin{tabular}{|c|c|c|}
\hline Nombre & $\begin{array}{l}\text { Compuesto / } \\
\text { Elemento }\end{array}$ & $\begin{array}{c}\text { Composición } \\
\text { (Wt \%) }\end{array}$ \\
\hline Sílice & $\mathrm{SiO}_{2}$ & 70,94 \\
\hline Aluminio & $\mathrm{Al}_{2} \mathrm{O}_{3}$ & 14,98 \\
\hline Hierro & $\mathrm{Fe}_{2} \mathrm{O}_{3}$ & 7,03 \\
\hline Potasio & $\mathrm{K}_{2} \mathrm{O}$ & 1,26 \\
\hline Titanio & $\mathrm{TiO}_{2}$ & 0,89 \\
\hline Bario & $\mathrm{BaO}$ & 0,15 \\
\hline Fosforo & $\mathrm{P}_{2} \mathrm{O}_{5}$ & 0,14 \\
\hline Magnesio & $\mathrm{MgO}$ & 0,05 \\
\hline Vanadio & $\mathrm{V}_{2} \mathrm{O}_{5}$ & 0,04 \\
\hline Zirconio & $\mathrm{ZrO}_{2}$ & 0,03 \\
\hline Estroncio & SrO & 0,03 \\
\hline Cromo & $\mathrm{Cr}_{2} \mathrm{O}_{3}$ & 0,01 \\
\hline Zinc & $\mathrm{ZnO}$ & 0,01 \\
\hline Calcio & $\mathrm{CaO}$ & ND \\
\hline Sodio & $\mathrm{Na}_{2} \mathrm{O}$ & ND \\
\hline Níquel & $\mathrm{NiO}$ & ND \\
\hline Cobre & $\mathrm{CuO}$ & ND \\
\hline Manganeso & $\mathrm{Mn}_{3} \mathrm{O}_{4}$ & ND \\
\hline Azufre & $\mathrm{SO}_{3}$ & ND \\
\hline $\begin{array}{l}\text { Perdida por } \\
\text { ignición }\end{array}$ & LOI & 4,9 \\
\hline
\end{tabular}

ND: No Detectable

Come se puede observar en la Tabla 1, la muestra está constituida en un $70,94 \%$ de Sílice, seguida por el Aluminio en forma de oxido con $14,98 \%$, esto concuerda con lo predicho en la teoría, porque la mayor parte de la composición de la arcilla se da por la presencia de Aluminosilicatos. Así mismo, la tabla indica que hay presencia de Hierro en forma Hematita en un $7,03 \%$, luego se encuentra el óxido de Potasio con $1,26 \%$. También hay presencia de minerales como Titanio, Fosforo y Bario en proporciones por debajo del $0,9 \%$. No se detecto presencia de minerales como Azufre, Cobre y Níquel.

El efecto de la difracción de rayos $X$ en la estructura mineral constituye la base para identificar los minerales $y$ sus sustancias cristalinas. Para la presente investigación, los espectros de DRX se registraron para la muestra de arcilla natural representativa a temperatura ambiente, los minerales asociados se identificaron utilizando el software XpertPro mediante las fichas de referencia (01-075-0443) Cuarzo, (01-075-0948) Moscovita, (01072-1673) Cronstedita y (01-074-1784) Caolinita.

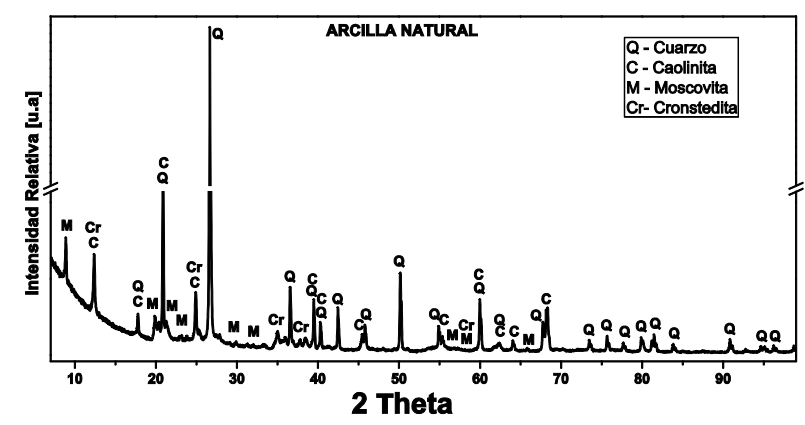

Figura 2. Difractograma de Rayos $X$ de la arcilla natural con fases mineralógicas mayoritarias identificadas.

El patrón de difracción DRX de la figura 2, muestra que los minerales de arcilla más abundantes en orden decreciente de acuerdo a la intensidad de los picos son: cuarzo, caolinita, moscovita y cronstedita. Mediante esta técnica no fue posible evidenciar fases con contenido de Fe en la arcilla natural.

\subsection{Identificación de fases de Fe en la arcilla natural y calentada.}

La información sobre la influencia de la temperatura y la atmósfera se obtiene partir de los parámetros Mössbauer como son: el corrimiento isómero (IS), el desdoblamiento cuadrupolar (QS), y las interacciones hiperfinas magnéticas las cuales especifican la presencia de Fe2+ / Fe3+y óxidos de Fe (Wagner et al., 2004). 


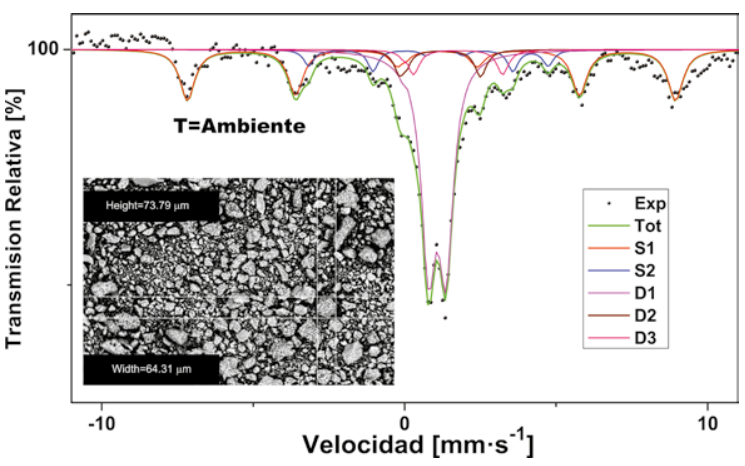

Figura 3. Espectro Mössbauer e imagen obtenida por SEM de la arcilla natural.

El espectro Mössbauer de la muestra de arcilla natural e imagen SEM de una fracción de muestra de la misma arcilla sin calentamiento artificial, se presenta en la figura 3. Se observa la presencia de óxido de Fe asociado a la hematita (S1), una segunda fase magnética (S2) con un campo magnético hiperfino de magnitud 292 KOe probablemente asociada a goethita y tres fases adicionales que comprenden los estados de oxidación en los cuales se encuentra el Fe en los minerales de la arcilla. Los resultados de los parámetros Mössbauer (corrimiento isomérico (IS ), desdoblamiento cuadrupolar (QS), campo magnético hiperfino $(H)$, ancho natural de línea a media altura (AHWL), área espectral relativa RA) derivados de la posición de los picos del espectro se presentan en la Tabla 2. Los valores de los parámetros hiperfinos obtenidos, indican que existen tres fases mayoritarias (hematita, $\mathrm{Fe}^{2+}$ y Fe${ }^{3+}$ ) en relación con las cinco fases inicialmente determinadas en la arcilla natural mediante EM.

De la morfología presentada en la imagen SEM de la figura 3 para la arcilla natural sin tratamiento térmico se observa que hay una distribución de tamaños no homogénea lo cual es un indicativo que los minerales de Fe pueden tener modos diferentes de agregación en la arcilla. Se establece que el tamaño de grano máximo para la arcilla sin calentar no supera las 80 micras y a medida que se calienta se observa un aumento en el tamaño de grano lo que origina agregados mayores que aportan a la creación de dominios magnéticos en minerales de hierro.

Al realizar el tratamiento térmico artificial de 400 a $800{ }^{\circ} \mathrm{C}$ a las muestras y tomar los espectros Mössbauer que se muestran en la figura 4 , se observa que el espectro de la muestra con calentamiento artificial a $400{ }^{\circ} \mathrm{C}$ comparada con el espectro registrado para la arcilla natural de la figura 3 , presenta una ganancia en la fase de hematita. Esto se debe posiblemente a que los iones de Fe presentes en las fases mineralógicas se oxidaron a medida que se incrementó la temperatura de calcinación aportando al oxido presente en la muestra.

Para la muestra con calentamiento artificial a $600{ }^{\circ} \mathrm{C}$ de la figura 4, el espectro muestra que la fase de hematita continua aumentando significativamente comparada con la muestra calentada a $400^{\circ} \mathrm{C}$ y la arcilla natural. También varía el área porcentual de las fases referentes al doblete central con respecto a la muestra de $400^{\circ} \mathrm{C}$, donde un doblete aumenta los valores del área porcentual de 5.43 a $7.46 \%$ y el otro doblete reduce su área porcentual pasando de 11.04 a $7.67 \%$ como se indica en la Tabla 2, lo que significa que la fase de Fe se oxida y aporta a la formación de la fase de hematita y el otro hace que aumente levemente la fase de $\mathrm{Fe} 2+$ con respecto a la arcilla calentada a $400^{\circ} \mathrm{C}$. Adicionalmente para esta muestra se observa un leve aumento del área espectral de la hematita (de 83.53 a $84.37 \%$ ) y del campo magnético (499.72 kOe). 


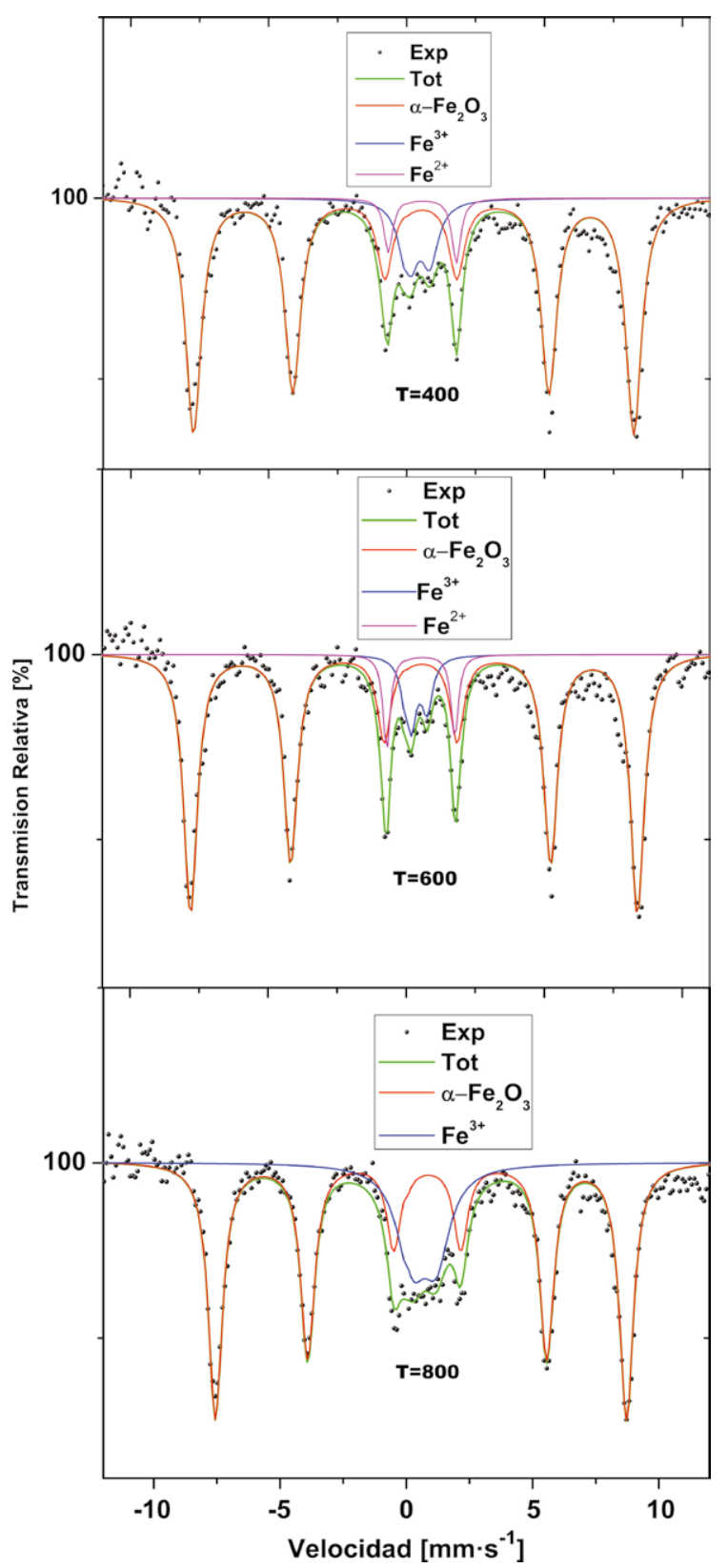

Figura 4. Espectros Mössbauer de la muestra de arcilla calentada a 400,600 y $800^{\circ} \mathrm{C}$

El espectro para la arcilla con tratamiento térmico artificial a $800^{\circ} \mathrm{C}$ de la figura 4 , muestra que uno de los dobletes se oxidó y el otro aumentó su área espectral, lo cual nos hace inferir que se crea un dominio donde los motivos atómicos del Fe se encuentran más ligados a la red. Con respecto al valor del campo magnético en el óxido de la hematita sigue aumentando su valor (502.794 kOe ver tabla 1), esto puede indicar que por encima de la temperatura de calentamiento de $600^{\circ} \mathrm{C}$ en la arcilla puede presentarse un ordenamiento magnético preferencial. Por otro lado se observa que permanece el doblete paramagnético en un estado de oxidación $\mathrm{Fe}^{3+}$.

Tabla 2. Parámetros Mössbauer (corrimiento isomérico (IS), desdoblamiento cuadrupolar (QS), campo magnético hiperfino $(H)$, ancho natural de línea a media altura (AHWL), área espectral relativa $R A$ ) de la muestra de arcilla natural (AN) y calentadas a 400,600,800, 1000 y $1200^{\circ} \mathrm{C}$

\begin{tabular}{|c|c|c|c|c|c|c|}
\hline Muestra & $\begin{array}{c}\text { IS } \\
{\left[\mathrm{mm} \cdot \mathrm{s}^{-1}\right]}\end{array}$ & $\begin{array}{c}\text { QS } \\
{\left[\mathrm{mm} \cdot \mathrm{s}^{-1}\right]}\end{array}$ & H(KOe) & $\begin{array}{c}\text { AHWL } \\
{\left[\mathrm{mm} \cdot \mathrm{s}^{-1}\right]}\end{array}$ & $\begin{array}{l}\text { RA } \\
\text { [\%] }\end{array}$ & FASE \\
\hline \multirow[t]{5}{*}{ AN } & 0.385 & -0.203 & 497 & 0.300 & 29.86 & Hematita \\
\hline & 0.717 & -0.264 & 292 & 0.206 & 7.93 & Goetita \\
\hline & 0.625 & 0.586 & 0 & 0.290 & 51.49 & $\mathrm{Fe}^{3+}$ \\
\hline & 1.103 & 2.652 & 0 & 0.233 & 5.58 & $\mathrm{Fe}^{2+}$ \\
\hline & 1.716 & 2.938 & 0 & 0.233 & 5.14 & $\mathrm{Fe}^{2+}$ \\
\hline \multirow[t]{3}{*}{$400^{\circ} \mathrm{C}$} & 0,387 & $-0,262$ & 492,881 & 0,342 & 83,53 & Hematita \\
\hline & 0,572 & 2,365 & 0 & 0,190 & 5,43 & $\mathrm{Fe}^{2+}$ \\
\hline & 0,431 & 0,730 & 0 & 0,364 & 11,04 & $\mathrm{Fe}^{3+}$ \\
\hline \multirow[t]{3}{*}{$600^{\circ} \mathrm{C}$} & 0,390 & $-0,241$ & 499,728 & 0,300 & 84,87 & Hematita \\
\hline & 0,401 & 0,591 & 0 & 0,238 & 7,67 & $\mathrm{Fe}^{3+}$ \\
\hline & 0,515 & 2,367 & 0 & 0,164 & 7,46 & $\mathrm{Fe}^{2+}$ \\
\hline \multirow[t]{2}{*}{$800^{\circ} \mathrm{C}$} & 0,687 & $-0,248$ & 502,794 & 0,320 & 79,37 & Hematita \\
\hline & 0,508 & 0,819 & 0 & 0,211 & 7,33 & $\mathrm{Fe}^{3+}$ \\
\hline \multirow[t]{3}{*}{$1000^{\circ} \mathrm{C}$} & 0,383 & $-0,272$ & 501,866 & 0,236 & 57,54 & Hematita \\
\hline & 0,401 & 0,754 & 0 & 0,250 & 29,09 & $\mathrm{Fe}^{3+}$ \\
\hline & 0,792 & 2,024 & 0 & 0,160 & 4,37 & $\mathrm{Fe}^{2+}$ \\
\hline \multirow[t]{2}{*}{$1200^{\circ} \mathrm{C}$} & 0,384 & $-0,264$ & 498,558 & 0,263 & 43,45 & Hematita \\
\hline & 0,341 & 1,028 & 0 & 0,303 & 56,55 & $\mathrm{Fe}^{3+}$ \\
\hline
\end{tabular}

En la figura 5 se muestra las imágenes SEM de las muestras de arcilla calentadas a $400^{\circ} \mathrm{C}, 600^{\circ} \mathrm{C}$ y $800^{\circ} \mathrm{C}$, en ellas se observa claramente un aumento en el tamaño de grano. Las imágenes $5 a, 5 b$ y $5 c$ muestran zonas brillantes que se pueden asociar a regiones donde se encuentra mayor contenido de materia mineral. En la imagen $5 c$ además se forman agregados de arcilla que probablemente por efecto de la temperatura han sufrido un proceso de deshidratación y deshidroxilacion produciendo un cambio morfológico y estructural en el cuerpo de la arcilla.

Rev. I2+D. Vol.16 N². Julio-Diciembre. 2016. Sogamoso-Boyacá, Colombia. 
A medida que la arcilla se va calentando a temperaturas que sobrepasan los $800^{\circ} \mathrm{C}$, se producen nuevas transformaciones en los minerales con contenido de Fe como se observa en la figura 6.

Para la temperatura a $1000^{\circ} \mathrm{C}$ el espectro muestra que la componente magnética presenta un cambio en el área espectral (@20\%) por la influencia de la temperatura en la muestra, es posible que a esta temperatura la arcilla ya no presente una estructura cristalina, sino una estructura irregular o amorfa que puede ocasionar que los momentos magnéticos que estaban fuertemente orientados se desorienten y en consecuencia haya una disminución del campo magnético hiperfino (ver Tabla 2, los valores cambian de 502.794 a 501.866 kOe). Asimismo, se presentan dos dobletes asociados a $\mathrm{Fe}^{2+}(\mathrm{D} 1)$ y $\mathrm{Fe}^{3+}(\mathrm{D} 2)$. El doblete de $\mathrm{Fe}^{3+}$ aumenta en proporción de área espectral, esto indica la presencia de un mineral de arcilla que aparece generalmente a altas temperaturas denominado mullita Häusler (2004).
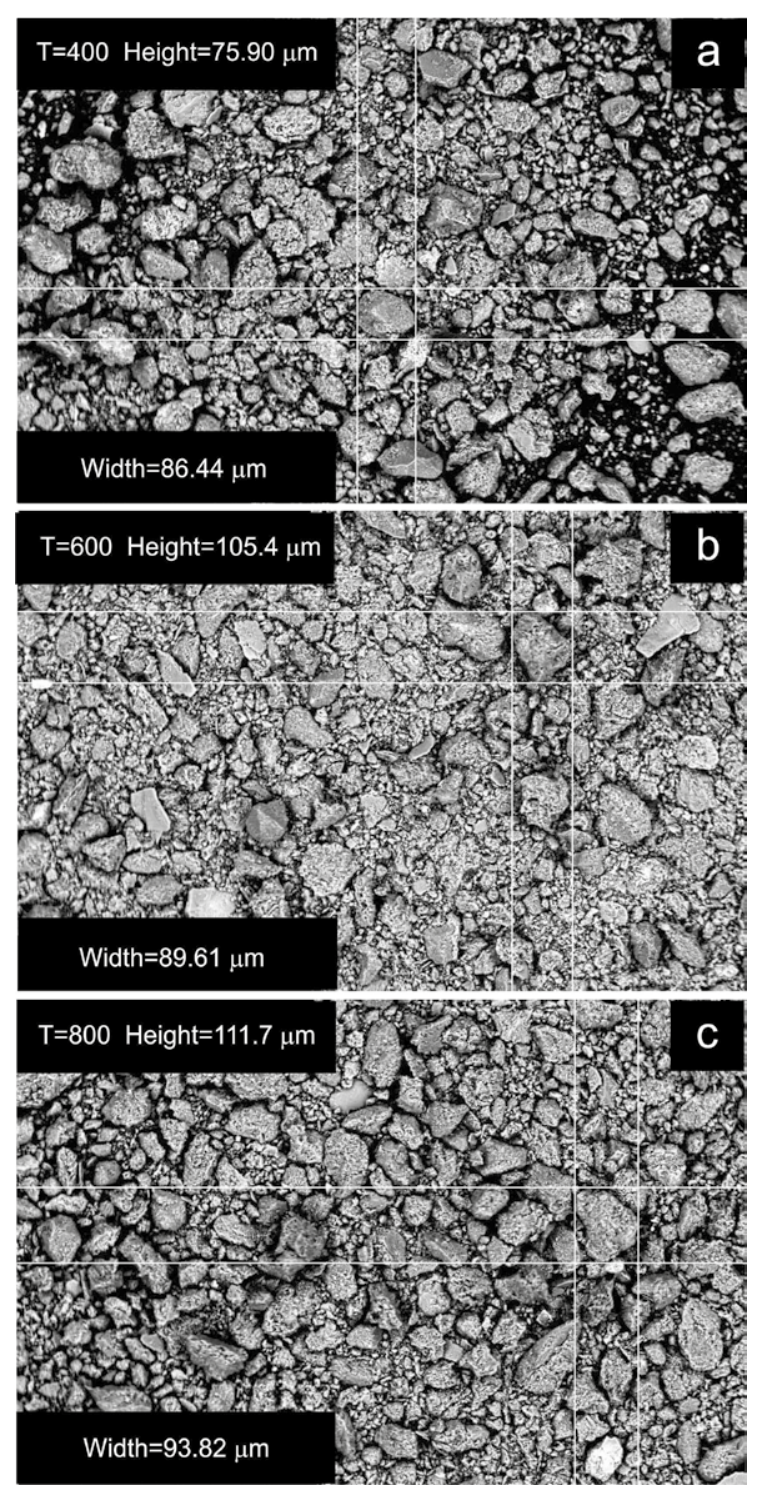

Figura 5. Imágenes SEM con aumento (200X) de las muestras de a. Arcilla calentada a $400^{\circ} \mathrm{C}$, b. Arcilla calentada a $600^{\circ} \mathrm{C}$, c. Arcilla calentada a $800^{\circ} \mathrm{C}$. 


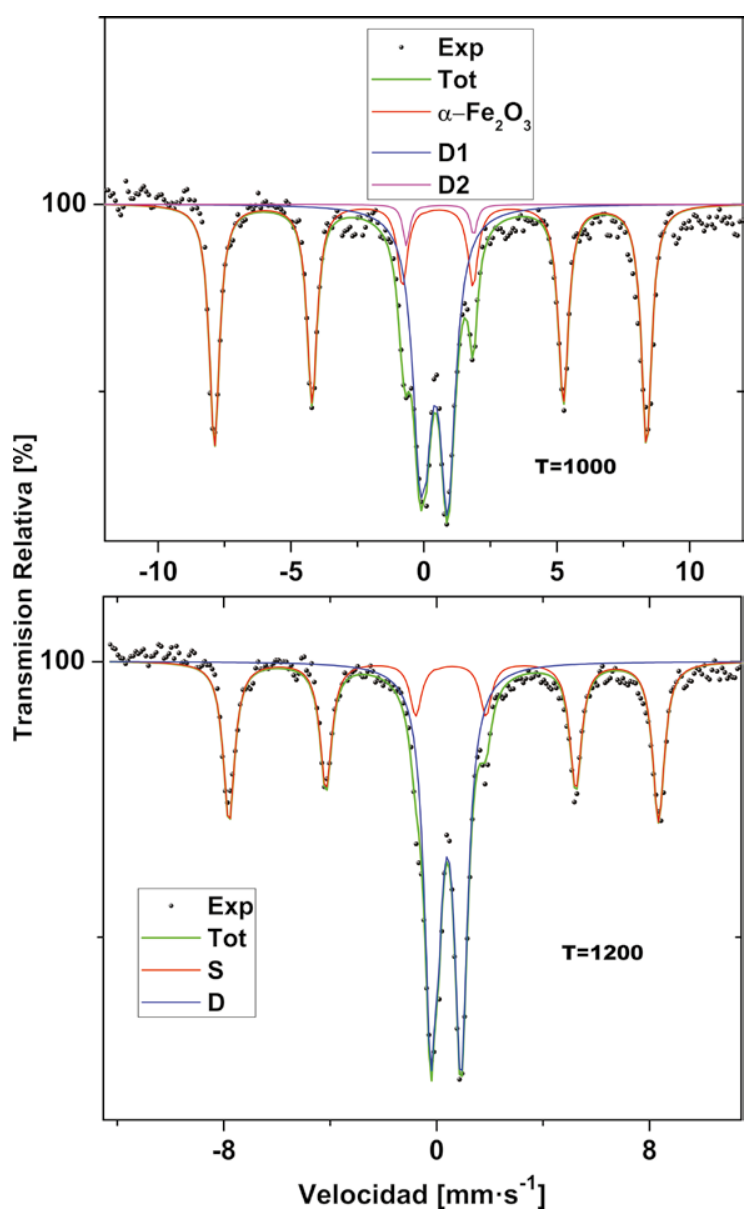

Figura 6. Espectros Mössbauer de la muestra de arcilla calentada a 1000 y $1200^{\circ} \mathrm{C}$

Para la muestra calentada hasta $1200{ }^{\circ} \mathrm{C}$ el espectro de la figura 6 indica que el valor del campo magnético disminuye (ver Tabla 2, su valor es $498.558 \mathrm{kOe}$ ) al igual que el área espectral (@10\%) con respecto al espectro de arcilla a $1000^{\circ} \mathrm{C}$. El espectro se ajustó tomando un sexteto que hace referencia a Hematita y un doblete asociado a la Mullita. La Mullita aumento su proporción en un valor de @ $14 \%$ con relación al espectro de $1000^{\circ} \mathrm{C}$. Los cambios observados a esta temperatura indican que existen transformaciones de los minerales dispuestos en la arcilla por encima de $1000^{\circ} \mathrm{C}$, específicamente de aquellas fases que presentan un contenido de $\mathrm{Fe}$ en su estructura cristalina.

La morfología de las muestras sometidas a tratamiento térmico de 1000 y $1200^{\circ} \mathrm{C}$ se muestra en la figura 7 .

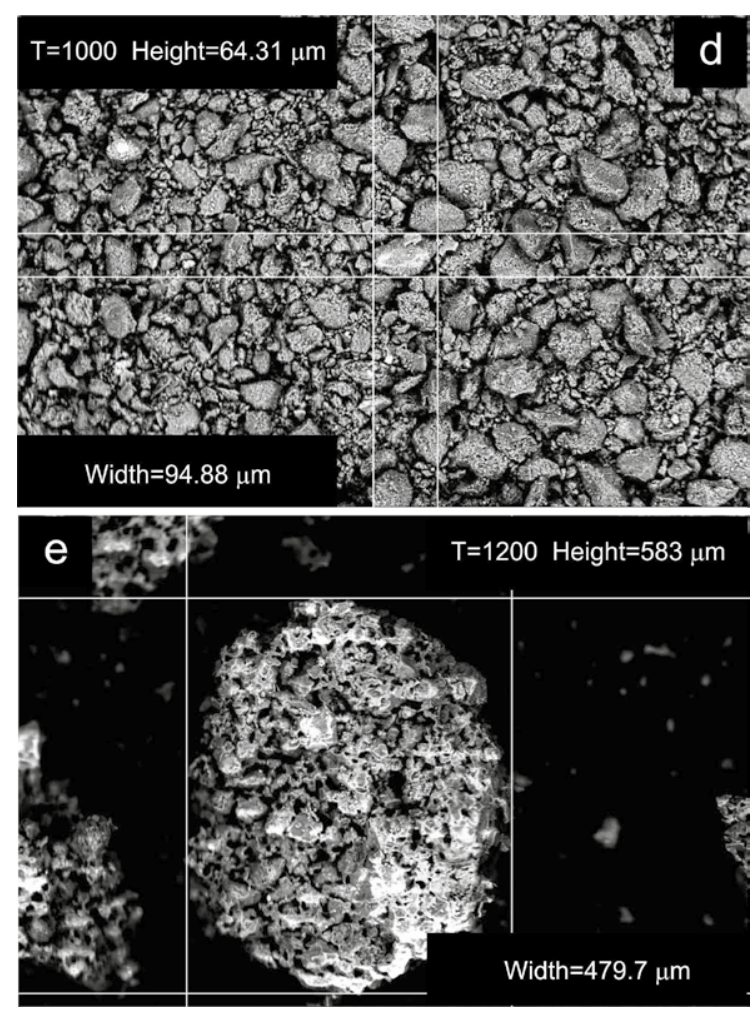

Figura 7. Imágenes SEM con aumento (200X) de las muestras de d. Arcilla calentada a $1000^{\circ} \mathrm{C}$, e. Arcilla calentada a $1200^{\circ} \mathrm{C}$.

Se evidencia claramente que a estas temperaturas el cuerpo de la arcilla cambia presentando un alto grado de densificación, lo cual da lugar a una posible cristalización donde se observan sitios en los cuales predomina la presencia de minerales con contenido de $\mathrm{Fe}$ (regiones brillantes del grano). Además, el tamaño de grano incremento aproximadamente en un factor de 10 con relación al tamaño de grano que presentó la arcilla natural.

Es de gran importancia resaltar que a medida que aumenta tratamiento térmico en la arcilla, los óxidos de Fe presentes y los cationes $\left(\mathrm{Fe}^{2+}, \mathrm{Fe}^{3+}\right)$ se transforman de modo que la fusión de estos últimos aportan a la oxidación de otros como es el caso particular de la hematita. Tales indicadores se pueden observar con claridad en los espectros Mössbauer de la arcilla natural y calentada a diferentes temperaturas $\left(400^{\circ} \mathrm{C}\right.$, $600^{\circ} \mathrm{C}, 800^{\circ} \mathrm{C}$. Por encima de $800^{\circ} \mathrm{C}$ se genera un efecto contrario, la hematita disminuye en proporción aportando al crecimiento en Mullita, mineral que en particular presenta estas características bien definidas a altas temperaturas. Por encima de los $1000{ }^{\circ} \mathrm{C}$ la arcilla

Rev. 12+D. Vol.16 N². Julio-Diciembre. 2016. Sogamoso-Boyacá, Colombia. 
Ruge-Guerrero, O.O., Martínez- Ovalle, S.A., \& Díaz-Lagos, M.

se ha recristalizado formando agregados de mayor tamaño que aportan a la reducción del comportamiento magnético presente en la hematita.

Los espectros Mössbauer de las muestras de arcilla natural sin calentamiento artificial y con calentamiento muestran un sexteto magnético y un doblete central. El campo magnético del sexteto aparece en torno a $51 \mathrm{~T}$ y el QS alrededor de $-0.20 \mathrm{~mm} \cdot \mathrm{s}^{-1}$ que son parámetros característicos de la hematita. Asimismo, se muestra una correlación con el aumento en la tonalidad del color del cuerpo de la arcilla con los resultados de los espectros Mössbauer de la arcilla calentada. La tonalidad rojiza se debe al óxido de Fe (hematita).

Es importante resaltar que el estudio se desarrolló en condiciones controladas de calentamiento y por esta razón se pudo identificar por medio de EM las transformaciones de los minerales de Fe dispuestos en la arcilla.

\section{REFERENCIAS}

Chevalier R., Coey J. M. D. \& Bouchez R. 1976. A study of iron in fired clay: Mössbauer effect and magnetic measurements. Journal de Physique: ques. 37(12): suppl., Colloq. 6, pp. C6.861-C6.865.

Gerstl Z. \& Banin A. 1980. $\mathrm{Fe}^{2+}-\mathrm{Fe}^{3+}$ transformations in clay and resin ion-exchange systems. Clays and Clay Minerals. 28, pp. 335-345. DOI: http://dx. doi.org/10.1346/CCMN.1980.0280503

Sanyal A. \& Mukerji S. J. 1986. Mössbauer study of the effect of iron on the synthesis of sialon from clay. Journal of Materials Science Letters. 5: pp. 787-788. DOI: http://dx.doi.org/10.1007/ BF01730092

Kreimeyer R. (1987). Some notes on the firing colour of clay bricks. Applied Clay Science. 2, pp. 175-183. DOI: http://dx.doi.org/10.1016/0169-1317(87)90007-X

Sherriff L. B., Mc Cammon C., Stirling L. 2002. A Mössbauer study of the color of roman pottery from the leptiminus archaeological site, tunisia. Geoarchaeology. 17(8), pp. 863-874. DOI: http://dx.doi.org/10.1002/gea.10044
Bernal I., Cabezas H. Espitia C. J. Mojica \& Quintero J. 2003. Análisis próximo de arcillas para cerámica. Rev. Academia Colombiana de Ciencias. 27(105), pp. 569-578.

Grygar D. T., Hradilova J., \& Bezdicka P. 2003. Clay and iron oxide pigments in the history of painting. Applied Clay Science. 22, pp. 223236. DOI: http://dx.doi.org/10.1016/S01691317(03)00076-0

Castelein O., Soulestin B., Bonnet J. P. \& Blanchart P. 2001. The influence of heating rate on the thermal behaviour and mullite formation from a kaolin raw material. Ceramics International. 27, pp. 517-522. DOI: http://dx.doi.org/10.1016/ S0272-8842(00)00110-3

Castelein O., Aldon I., Olivier-fourcade J., Jumas J. C., Bonnet J. P. \& Blanchart P. 2002. ${ }^{57} \mathrm{Fe}$ Mössbauer study of iron distribution in a kaolin raw material: influence of the temperature and the heating rate. Journal of the European Ceramic Society. 22(11), pp. 1767-1773.

Häusler W. 2004. Firing of clays studied by x-ray diffraction and Mössbauer spectroscopy. Hyperfine interactions. 154, pp. 121-141. DOI: http://dx.doi. org/10.1023/B:HYPE.0000032075.92893.57

Murad E. 2006. Mössbauer spectroscopy of clays and clay minerals. Developments in Clay Science. 1, pp. 755-764. DOI: http://dx.doi.org/10.1016/ S1572-4352(05)01027-5

Abdrakhimova E. S. \& Abdrakhimov V. Z. 2006. A Mössbauer spectroscopy study of the transformation of iron compounds in clay materials. Russian Journal of Physical Chemistry. 80(7), pp. 1077-1082. DOI: http://dx.doi.org/10.1134/ S0036024406070132

Calogero S., Bertelle M., Antonelli M. \& Lazzarini L. 2000. A Mössbauer study of some coloured marbles (Cipollino Mandolato, Rosso Antico and Fior Di Pesco): implications on the nature of their colour. Journal of Cultural Heritage. 1 , pp. 429-444. DOI: http://dx.doi.org/10.1016/ S1296-2074(00)01098-0 
Dionísio A. M., Sequeira A. \& Waerenborgh J. C. 2009. Clay minerals and iron oxides-oxyhydroxides as fingerprints of firing effects in a Limestone monument. Applied Clay Science. 42, pp. 629-638. DOI: http://dx.doi.org/10.1016/j. clay.2008.05.003

Valanciene V., Siauciunas R. \& Baltusnikaite J. 2010. The influence of mineralogical composition on the colour of clay body. Journal of the European Ceramic Society. 30, pp. 1609-1617. DOI: http://dx.doi.org/10.1016/j.jeurceramsoc.2010.01.017

Orolínová Z., Mockovciaková A., Zelenák V. \& Myndyk M. 2012. Influence of heat treatment on phase transformation of clay-iron oxide composite. Journal of Alloys and Compounds. 511, pp. 63- 69. DOI: http://dx.doi.org/10.1016/j.jallcom.2011.08.023

Munsell, A. (2016).Recuperado www.munsell.com

Kamseu E., Leonelli C., Boccaccini D.N., Veronesi P., Miselli P., Pellacani G. \& Chinje Melo U. 2007. Characterization of porcelain compositions using t wo china clays from Cameroon. Ceramics International. 33, pp. 851-857. DOI: http://dx.doi. org/10.1016/j.ceramint.2006.01.025
Naranjo W., Gaviria S., Manosalva S. 2007. Mineralogía y Geoquímica de Diatomitas (Boyacá, Colombia). Geología Colombiana. 32, pp. 77-88.

Njoya D., Hajjaji, M., Bacaoui A. \& Njopwouo D. 2010. Microstructural characterization and influence of manufacturing parameters on technologi$c$ al properties of vitreous ceramic materials. $\mathrm{M}$ aterials Characterization. 61(3), pp. 2892 95. DOI: http://dx.doi.org/10.1016/j.matchar.2009.12.009

D jangang N.C., Elimbi A., Melo U.C., Lecomte G.L., N koumbou C., Soro J., Yvon J., Blanchart P. \& Njopwouo, D. 2008. Refractory ceramics from c lays of Mayouom and Mvan in Cameroon. Applied Clay Science. 39, pp. 10-18. DOI: http:// dx.doi.org/10.1016/j.clay.2007.04.006

D jangang N.C., Kamseu E., Ndikontar K.R. Lecomte N ana G.L., Soro J., Chinje Melo U., Elimbi A., Blanchart P., Njopwouo D. 2011. Sintering be$h$ aviour of porous ceramic kaolincorundum composites: phase evolution and densification. Materials Science and Engineering A. 528, pp. 8 311-8318. DOI: http://dx.doi.org/10.1016/j. msea.2011.07.006 